(1)

CrossMark

\title{
Increase in circulating ACE-positive endothelial microparticles during acute lung injury
}

\author{
Yusuke Takei ${ }^{1}$, Mitsuhiro Yamada $\mathbb{1}^{2}$, Koji Saito ${ }^{3}$, Yoshinobu Kameyama ${ }^{3}$, \\ Hisatoshi Sugiura², Tomonori Makiguchi², Naoya Fujino², Akira Koarai², \\ Hiroaki Toyama', Kazutomo Saito', Yutaka Ejima', Yu Kawazoe ${ }^{4}$, Daisuke Kudo ${ }^{4}$, \\ Shigeki Kushimoto ${ }^{4}$, Masanori Yamauchi ${ }^{1}$ and Masakazu Ichinose ${ }^{2}$
}

Affiliations: ${ }^{1}$ Dept of Anaesthesiology and Perioperative Medicine, Tohoku University Graduate School of Medicine, Sendai, Japan. ${ }^{2}$ Dept of Respiratory Medicine, Tohoku University Graduate School of Medicine, Sendai, Japan. ${ }^{3}$ Dept of Intensive Care Unit, Tohoku University Graduate School of Medicine, Sendai, Japan. ${ }^{4}$ Division of Emergency and Critical Care Medicine, Tohoku University Graduate School of Medicine, Sendai, Japan.

Correspondence: Mitsuhiro Yamada, Dept of Respiratory Medicine, Tohoku University Graduate School of Medicine, 1-1 Seiryo-machi, Aoba-ku, Sendai, Miyagi 9808574, Japan. E-mail: yamitsulamed.tohoku.ac.jp

@ERSpublications

Circulating $\mathrm{ACE}^{+}$endothelial microparticles from pulmonary microvascular endothelial cells could be a marker for evaluating the injury of pulmonary microvascular endothelial cells and a prognostic marker for developing ARDS in septic patients http://bit.ly/2GeX7ap

Cite this article as: Takei Y, Yamada M, Saito K, et al. Increase in circulating ACE-positive endothelial microparticles during acute lung injury. Eur Respir J 2019; 54: 1801188 [https://doi.org/10.1183/ 13993003.01188-2018]

ABSTRACT Circulating endothelial microparticles (EMPs) are considered to be markers of endothelial injury, and lung microvascular endothelial cells express higher levels of angiotensin-converting enzyme (ACE). The aim of this study is to examine whether the number of $\mathrm{ACE}^{+}$microvascular EMPs could be a prognostic marker for the development of acute respiratory distress syndrome (ARDS) in septic patients.

The numbers of EMPs and $\mathrm{ACE}^{+}$EMPs in the culture supernatant from human microvascular endothelial cells, as well as in the blood of mouse lung injury models and septic patients $(n=82)$, were examined using flow cytometry.

$\mathrm{ACE}^{+}$EMPs in the culture supernatant from pulmonary microvascular endothelial cells increased after exposure to an inflammatory stimulus. In the mouse lung injury models, the circulating $\mathrm{ACE}^{+} \mathrm{EMPs}$ and $\mathrm{ACE}^{+} \mathrm{EMP} / \mathrm{EMP}$ ratio were higher than in the controls $(\mathrm{p}<0.001)$. The $\mathrm{ACE}^{+} \mathrm{EMP} / \mathrm{EMP}$ ratio was correlated with the wet/dry lung ratio $\left(\mathrm{r}_{\mathrm{s}}=0.775, \mathrm{p}<0.001\right)$. The circulating $\mathrm{ACE}^{+} \mathrm{EMPs}$ and $\mathrm{ACE}^{+} \mathrm{EMP} /$ EMP ratio on admission were significantly increased in septic patients who developed ARDS compared with septic patients who did not $(\mathrm{p}<0.001)$.

Therefore, circulating $\mathrm{ACE}^{+}$EMPs may be a prognostic marker for the development of ARDS in the septic patients. 


\section{Introduction}

Microparticles are extracellular vesicles with dimensions between $0.1 \mu \mathrm{m}$ and $1.0 \mu \mathrm{m}[1,2]$. Microparticles bud off from the plasma membrane during injury, activation, apoptosis, pyroptosis or necrosis $[3,4]$. Recent reports have suggested that microparticles participate in intercellular communication $[1,3,5]$.

It has been reported that the number of endothelial microparticles (EMPs; microparticles derived from endothelial cells) increases in diseases accompanied by endothelial injury such as acute coronary syndrome, renal failure and chronic obstructive pulmonary disease [6-12]. Furthermore, these reports suggested that the number of circulating EMPs reflects the extent of endothelial injury and disease severity [8].

Acute respiratory distress syndrome (ARDS) consists of non-cardiac pulmonary oedema with increased pulmonary permeability caused by extensive invasive disease, such as sepsis or severe pneumonia $[13,14]$. The pathological changes in alveoli during ARDS involve disruption of the alveolar-capillary barrier due to lung epithelial and endothelial injuries $[15,16]$. On this basis, we propose that EMPs could be a marker of pulmonary endothelial injury and predict ARDS in patients at risk. However, diseases causing ARDS, such as sepsis, provoke severe systemic inflammation, resulting in systemic vascular injury. Therefore, analysing EMPs that express a specific antigen from the pulmonary microvascular endothelium is critical to distinguish pulmonary microvascular injury from systemic capillary injury and to utilise EMPs as biomarkers of ARDS. We focused on angiotensin-converting enzyme (ACE), a zinc-dependent metalloprotease present on the cell [17]. Pulmonary microvascular endothelial cells express higher levels of ACE compared with other endothelial cells, including the systemic capillary cells [18-20]. Therefore, we hypothesised that circulating $\mathrm{ACE}^{+}$EMPs would increase in mouse lung injury models and in septic patients who develop ARDS.

First, we analysed whether $\mathrm{ACE}^{+}$EMPs were released from pulmonary microvascular endothelial cells and whether the budding of $\mathrm{ACE}^{+}$EMPs increased after exposure to an inflammatory stimulus. Then, we examined changes in $\mathrm{ACE}^{+} \mathrm{EMPs}$ and their correlation with the severity of lung injury in mouse injury models. We further examined whether the number of $\mathrm{ACE}^{+} \mathrm{EMPs}$ was significantly increased in septic patients who developed ARDS compared with septic patients who did not.

Materials and methods

Detailed information is provided in the supplementary material.

\section{Enumeration of $\mathrm{ACE}^{+}$EMPs in culture supernatant}

Human pulmonary microvascular endothelial cells (HPMECs), human dermal blood endothelial cells (HDBECs) and human pulmonary artery endothelial cells (HPAECs) were purchased from PromoCells (Heidelberg, Germany). The cells were incubated with the indicated stimuli for $24 \mathrm{~h}$. The supernatant was analysed using flow cytometry to count $\mathrm{CD}^{+} 1^{+}$EMPs and $\mathrm{ACE}^{+} \mathrm{CD} 31^{+}$EMPs.

\section{Enumeration of circulating $\mathrm{ACE}^{+}$EMPs in mouse lung injury models}

All experimental protocols were approved by the animal care committee at the Tohoku University School of Medicine (Sendai, Japan). 8-12-week-old C57BL/6J male mice were used. Lung injury was induced by caecal ligation and puncture (CLP) for the indirect injury model $[21,22]$ or by intratracheal lipopolysaccharide (LPS) administration for the direct injury model [23, 24]. Blood samples for the EMP analysis were obtained via inferior vena cava puncture $24 \mathrm{~h}$ after the procedure. The lungs were fixed by intratracheal instillation of $4 \%$ paraformaldehyde at $20 \mathrm{cmH}_{2} \mathrm{O}$ for the histological evaluation of lung injury [25]. In addition, the wet/dry lung (W/D) ratio was measured for alterations in pulmonary permeability [26].

\section{Enumeration of circulating ACE EMPs in septic patients}

The prospective cohort study was performed to examine circulating $\mathrm{ACE}^{+}$EMPs in septic patients (figure 1; more detail given in the supplementary material). This study was approved by the Tohoku University School of Medicine ethics committee (2015-1-279). The subjects were admitted to the intensive care unit or medical emergency centre at Tohoku University Hospital. Sepsis was diagnosed according to the Third International Consensus Definitions for Sepsis and Septic Shock (Sepsis-3) [27] and ARDS was diagnosed according to the Berlin definition [13]. Written informed consent was obtained from the patient's next of kin before recruitment. Blood samples were drawn to prepare serum at the time of inclusion. Patients were followed for 5 days for development of ARDS.

\section{Statistical analysis}

The data are expressed as median (interquartile range), as indicated. Statistical tests were performed using GraphPad Prism 7.0 (GraphPad Software, San Diego, CA, USA) or JMP Pro 13.2.1 (SAS, Cary, NC, USA). 


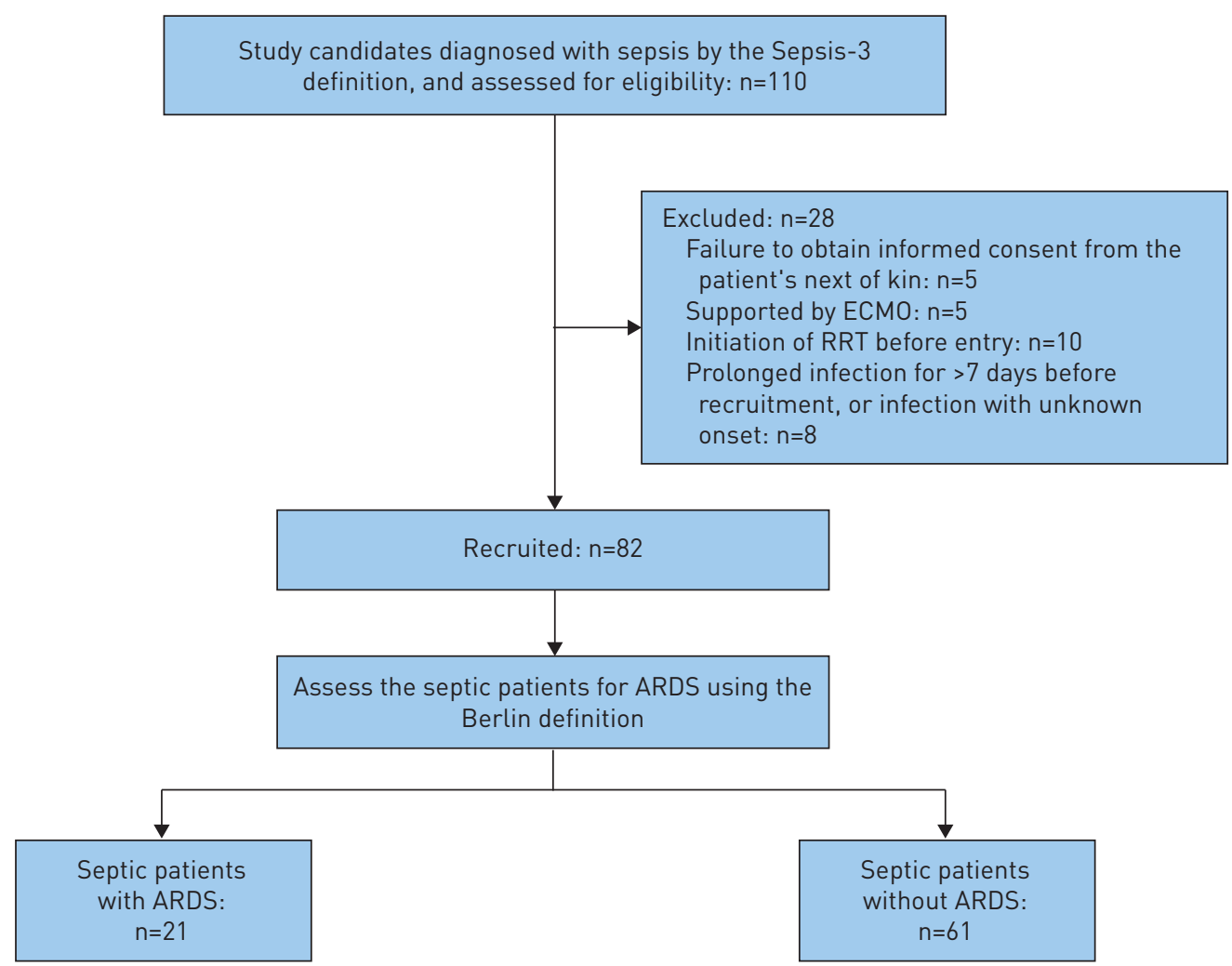

FIGURE 1 Profile of the prospective cohort study to examine circulating angiotensin-converting enzyme (ACE) ${ }^{+}$ endothelial microparticles in septic patients. Sepsis-3: Third International Consensus Definitions for Sepsis and Septic Shock; ECMO: extracorporeal membrane oxygenation; RRT: renal replacement therapy; ARDS: acute respiratory distress syndrome.

Statistical analyses were assessed by Wilcoxon rank sum tests or t-tests ( $\mathrm{n}=3$ for each group) to compare variables between two groups. For comparisons among more than three groups, the Kruskal-Wallis test was used, followed by Dunn's test. Statistical correlation analyses were performed using Spearman's test. Probability values $<0.05$ were considered significant.

\section{Results}

An inflammatory stimulus induced the formation of ACE ${ }^{+}$EMPs from pulmonary microvascular endothelial cells

To evaluate the possibility of $\mathrm{ACE}^{+}$EMPs being a marker of pulmonary vascular injury, we examined whether exposure to an inflammatory stimulus induced the formation of $\mathrm{ACE}^{+} \mathrm{EMPs}$ from pulmonary microvascular endothelium. We first examined the expression of ACE on the human primary endothelial cells (supplementary figure S1a). The percentage of $\mathrm{ACE}^{+}$cells (supplementary figure S1b) and the ACE intensity (supplementary figure S1c) were significantly higher in HPMECs than in HDBECs (as representatives of systemic microvascular endothelial cells) or HPAECs. These data suggested that pulmonary microvascular cells expressed higher levels of ACE than systemic microvascular cells or pulmonary artery endothelial cells, as previously reported [18-20]. Exposure to an inflammatory stimulus (LPS or tumour necrosis factor (TNF)- $\alpha$ ) induced a significant decrease in the expression of ACE both on HPMECs and HDBECs (supplementary figure S1e-g).

We then examined the ACE expression on EMPs in the culture supernatant from HPMECs or HDBECs. We set the gate for microparticles using a mix of fluorescent beads of various diameters during flow cytometry (supplementary figure S2) [28]. No CD31 ${ }^{+}$EMPs in the culture supernatant samples were detected by flow-cytometry after treating these samples with Triton X-100 (supplementary figure S7a), suggesting that the EMPs detected by flow cytometry were lipid membrane-bound vesicles. In addition, these EMPs were stained with PKH26, a cell membrane labelling reagent, also suggesting that these EMPs were lipid-membrane bound vesicles (supplementary figure S7b). Some EMPs in the culture supernatant are positive for CD9, a marker for exosomes (supplementary figure S7c). 
a)

HPMEC-sup
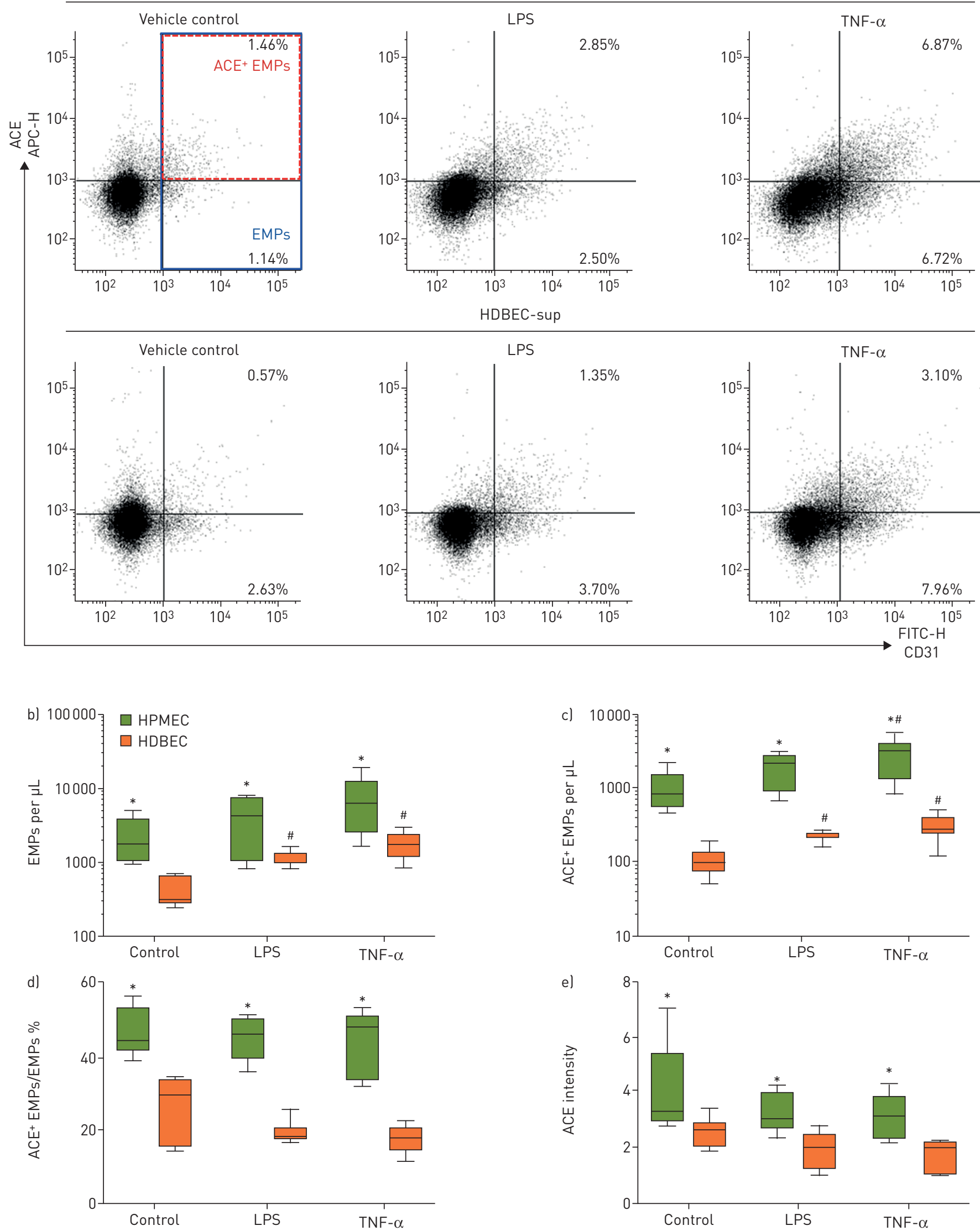
FIGURE 2 Exposure to an inflammatory stimulus induces an increase in angiotensin-converting enzyme (ACE) highly expressing endothelial microparticles (EMPs) released from human pulmonary microvascular endothelial cells. a) Representative images of flow cytometric analyses of EMPs in culture supernatants. Upper panels show images of culture supernatants of human pulmonary microvascular endothelial cells (HPMECs). Lower panels show images of culture supernatants of human dermal blood endothelial cells (HDBECs). Left panels show vehicle (PBS) controls. Centre panels show culture supernatants stimulated with lipopolysaccharide (LPS) (100 ng. $\mathrm{mL}^{-1}$ ). Right panels show culture supernatants stimulated with tumour necrosis factor (TNF)- $\alpha\left(25 \mathrm{ng} \cdot \mathrm{mL}^{-1}\right)$. Note that EMPs are identified as CD31-positive microparticles (within the blue line square). ACE ${ }^{+}$EMPs are identified as CD31-positive and ACE-positive microparticles (within the red dashed line square). The counts of b) EMPs and c) ACE ${ }^{+}$EMPs in culture supernatants of HPMECs or HDBECs. d) The ratio (\%) of ACE EMPs to total EMPs (ACE ${ }^{+}$EMP/EMP ratio). e) The geometric mean of the ACE expression levels normalised by the corresponding isotype control antibody staining on HPMECs or HDBECs. $\mathrm{n}=6$ for each experimental group. Box and whisker plots show the first and third quartiles, the median, and the minimum and maximum. *: $p<0.05$ versus HDBEC, ${ }^{\#}$ : $p<0.05$ versus each control. APC: allophycocyanin; FITC: fluorescein isothiocyanate; sup: supernatant.

There were significantly more total EMPs and $\mathrm{ACE}^{+}$EMPs in the supernatant from HPMECs, whether exposed or not exposed to an inflammatory stimulus, than in supernatant from HDBECs (figure $2 \mathrm{~b}$ and $\mathrm{c}$ ). An inflammatory stimulus increased EMPs and $\mathrm{ACE}^{+}$EMPs in the culture supernatant from both types of endothelial cells.

The $\mathrm{ACE}^{+} \mathrm{EMP} / \mathrm{EMP}$ ratio and the intensity of ACE on EMPs were significantly higher in the supernatant from HPMECs than in the supernatant from HDBECs regardless of the exposure to stimuli (figure $2 \mathrm{~d}$ and $\mathrm{e}$ ). These results suggest that inflammatory stimuli induce the formation of $\mathrm{ACE}^{+}$EMPs (EMPs highly expressing ACE) from pulmonary microvascular endothelial cells, resulting in decreased levels of cell-surface ACE expression.

It has been reported that p38 mitogen-activated protein kinase (MAPK) activation, caspase-dependent cell death (apoptosis or pyroptosis) or necrosis are involved in the formation of EMPs after exposure to inflammatory stimuli $[3,29,30]$. We investigated which process contributed to the formation of EMPs from HPMECs using pharmacological inhibitors. Stimulation with TNF- $\alpha$ increased annexin V-positive cells in HPMECs (supplementary figure S3a and b). Only Z-VAD-FMK, a pan-caspase inhibitor, but not SB203850 (p38 MAPK inhibitor) and necrostatin-1 (necroptosis inhibitor), attenuated the increases in annexin V-positive cells, suggesting that Z-VAD-FMK inhibited the apoptosis of HMPECs (supplementary figure S3a and b). Stimulation with TNF- $\alpha$ increased EMPs including annexin V-positive EMPs in the supernatant of HPMECs. Z-VAD-FMK, but not SB203850 or necrostatin-1, inhibited the increases in EMPs and annexin V-positive EMPs (supplementary figure S3c-h). These findings suggest that significant amounts of EMPs induced by TNF- $\alpha$ stimulation were possibly derived from apoptosis or pyroptosis which was inhibited by Z-VAD-FMK.

\section{Circulating $\mathrm{ACE}^{+}$EMPs increased in mouse lung injury models and possibly correlated with the severity of lung injury}

Because inflammatory stimulation induces the budding of $\mathrm{ACE}^{+}$EMPs from pulmonary microvascular endothelium, we hypothesised that the circulating $\mathrm{ACE}^{+} \mathrm{EMP}$ numbers would increase during acute lung injury, which involves injury of the pulmonary microvasculature, and the $\mathrm{ACE}^{+} \mathrm{EMP}$ numbers correlate with the severity of lung injury. To address this issue, we utilised different types of injury models, including the CLP-induced injury model (CLP model; as a model of indirect injury) and the intratracheally administered LPS-induced injury model (LPS-IT model; as a model of direct injury).

Histological analyses showed the infiltration of neutrophils in the alveolar interstitium and thickened alveolar septa in the CLP-model mice, whereas the LPS-IT-model mice showed the infiltration of neutrophils in the alveolar space (supplementary figure S4a-d). The lung injury scores (supplementary figure S4e) and W/D ratio (supplementary figure S4f) of the models were significantly increased compared with the corresponding controls.

We examined circulating EMPs in the injury models using flow cytometry. Total EMP numbers were significantly increased compared with the respective controls (figure $3 \mathrm{a}$ ). $\mathrm{ACE}^{+} \mathrm{EMP}$ numbers were significantly increased in injury models (figure $3 \mathrm{~b}$ ). Moreover, the $\mathrm{ACE}^{+} \mathrm{EMP} / \mathrm{EMP}$ ratio significantly increased in the injury models compared to the respective controls (figure 3c), indicating that the increase in the counts of $\mathrm{ACE}^{+}$EMPs in the injury models was not due to the increase in total EMPs, but probably due to the increase in the $\mathrm{ACE}^{+} \mathrm{EMP}$ numbers released from lung microvascular endothelium.

We analysed the correlation between the circulating EMPs and the severity of lung injury. The counts of circulating EMPs had a moderate correlation with the W/D ratio $\left(r_{s}=0.635, p<0.001\right)$. ACE EMPs $^{+}$ $\left(r_{s}=0.812, p<0.001\right)$ and the $A_{C E}^{+}$EMP/EMP ratio $\left(r_{s}=0.775, p<0.001\right.$; figure $\left.3 d\right)$ had a stronger correlation with the W/D ratio than with the total EMPs. The CLP-model mice showed variable W/D ratios, even in the same experimental group that underwent the same procedure. This variability may be due to the caecal ligation length, which is a major factor in the severity in CLP, and was different because 

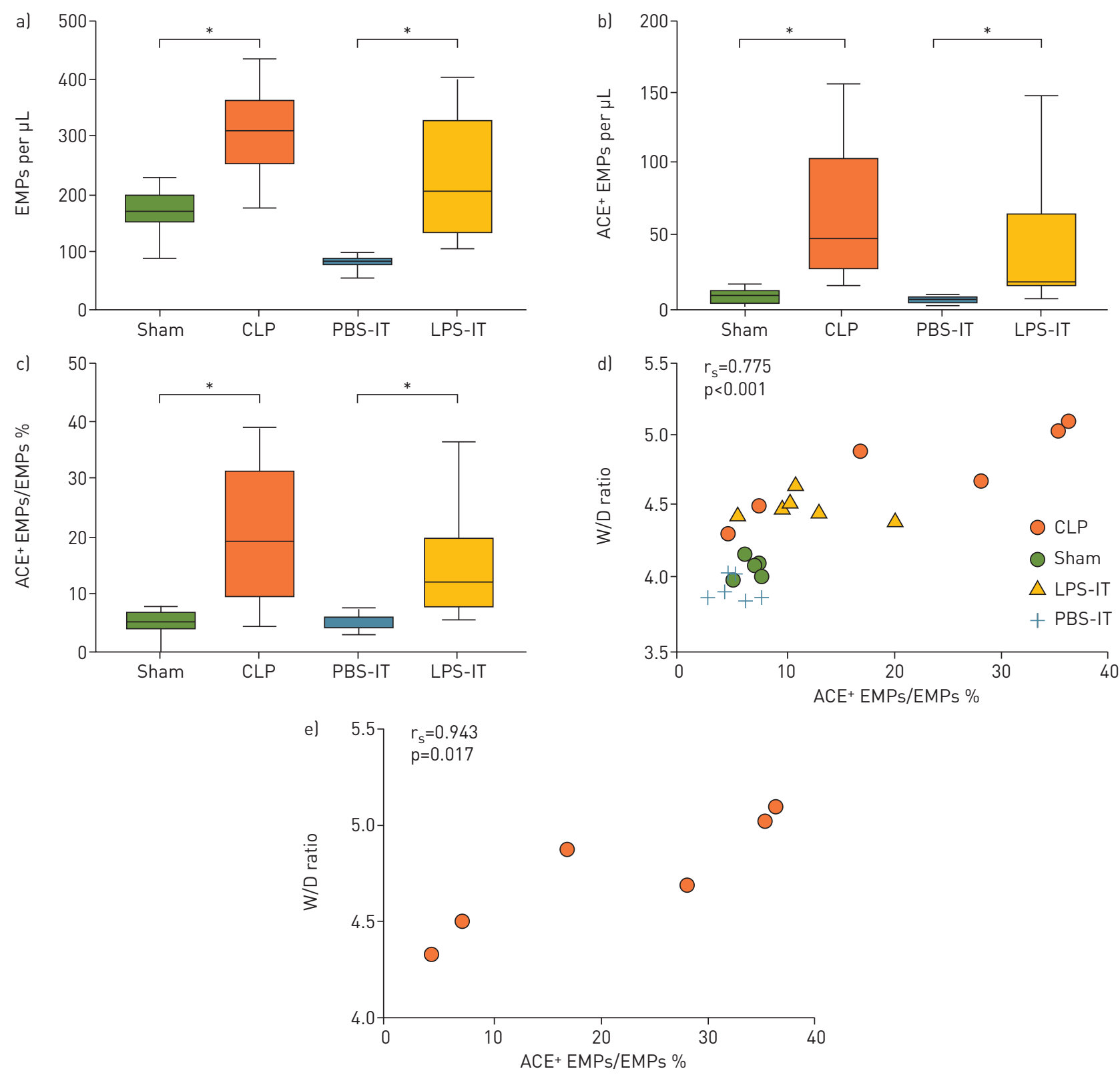

FIGURE 3 Circulating angiotensin-converting enzyme (ACE) ${ }^{+}$endothelial microparticles (EMPs) increased in both lung injury models, and this increase was correlated with the changes in lung permeability. The counts of a) EMPs, b) ACE EMPs and c) the ACE ${ }^{+}$EMP/EMP ratio in sham-operated mice (sham), caecal ligation and puncture (CLP)-operated mice, intratracheal PBS-administered mice (PBS-IT) and intratracheal lipopolysaccharide (LPS)-administered mice (LPS-IT; $n=12$ in each group); d) the correlation between the ACE ${ }^{+}$EMP/EMP ratio and the wet/dry lung (W/D) ratio in all mice ( $n=6$ in each group); e) the correlation between the ACE ${ }^{+}$EMP/EMP ratio and the W/D ratio in CLP-operated mice ( $n=6$ in each group). Box and whisker plots show the first and third quartiles, the median and the minimum and maximum. *: $\mathrm{p}<0.05$ between the two groups. $r_{\mathrm{s}}$ : Spearman's rank correlation coefficient.

the individual caecum size varies. Interestingly, their $\mathrm{ACE}^{+} \mathrm{EMP} / \mathrm{EMP}$ ratios were also significantly correlated with the W/D ratio $\left(\mathrm{r}_{\mathrm{s}}=0.943, \mathrm{p}=0.017\right.$; figure 3e). These data suggested that circulating $\mathrm{ACE}^{+}$ EMPs and the $\mathrm{ACE}^{+}$EMP/EMP ratio possibly reflected the severity of lung injury, including alterations in the alveolar-capillary barrier.

The immunofluorescent staining of the section of mouse lungs showed that $\mathrm{CD}_{3} 1^{+}$alveolar endothelial cells expressed ACE (supplementary figure S5). The immunohistochemical examination of ACE suggested that the intensity of ACE staining probably decreased in the alveoli of the CLP-model mice (figure 4a). Furthermore, Western blotting analyses showed that the levels of ACE decreased in whole lungs of the injured mice, whereas the levels of ACE protein in other systemic organs, both liver and kidney, were not significantly changed in the CLP animals (figure $4 \mathrm{~b}$ and c). In addition, we performed isolation of the 
a)
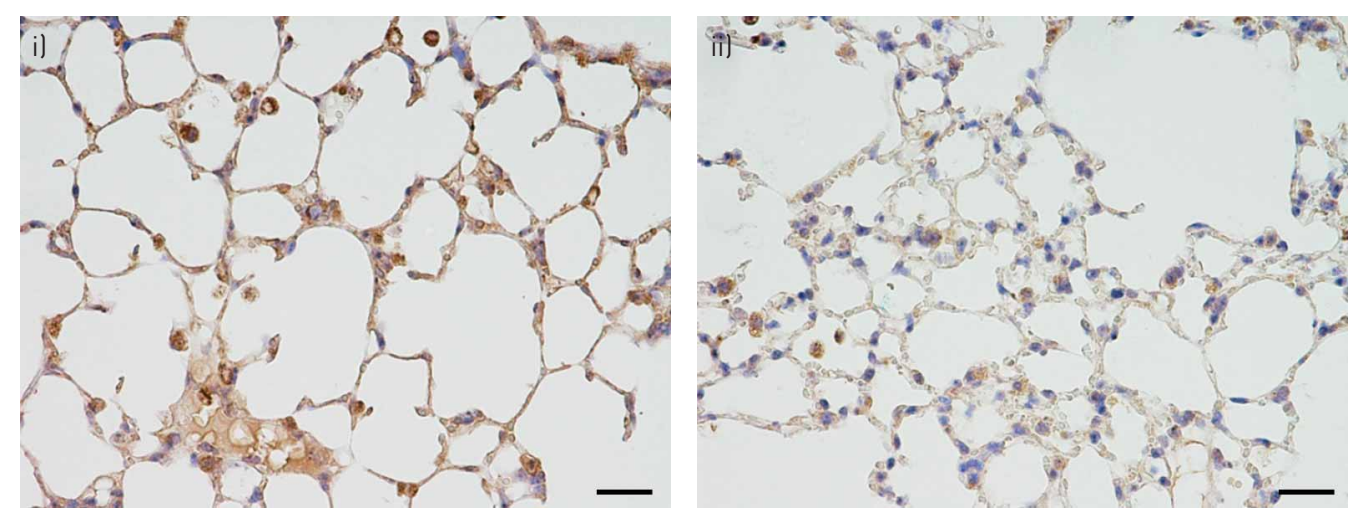

b)
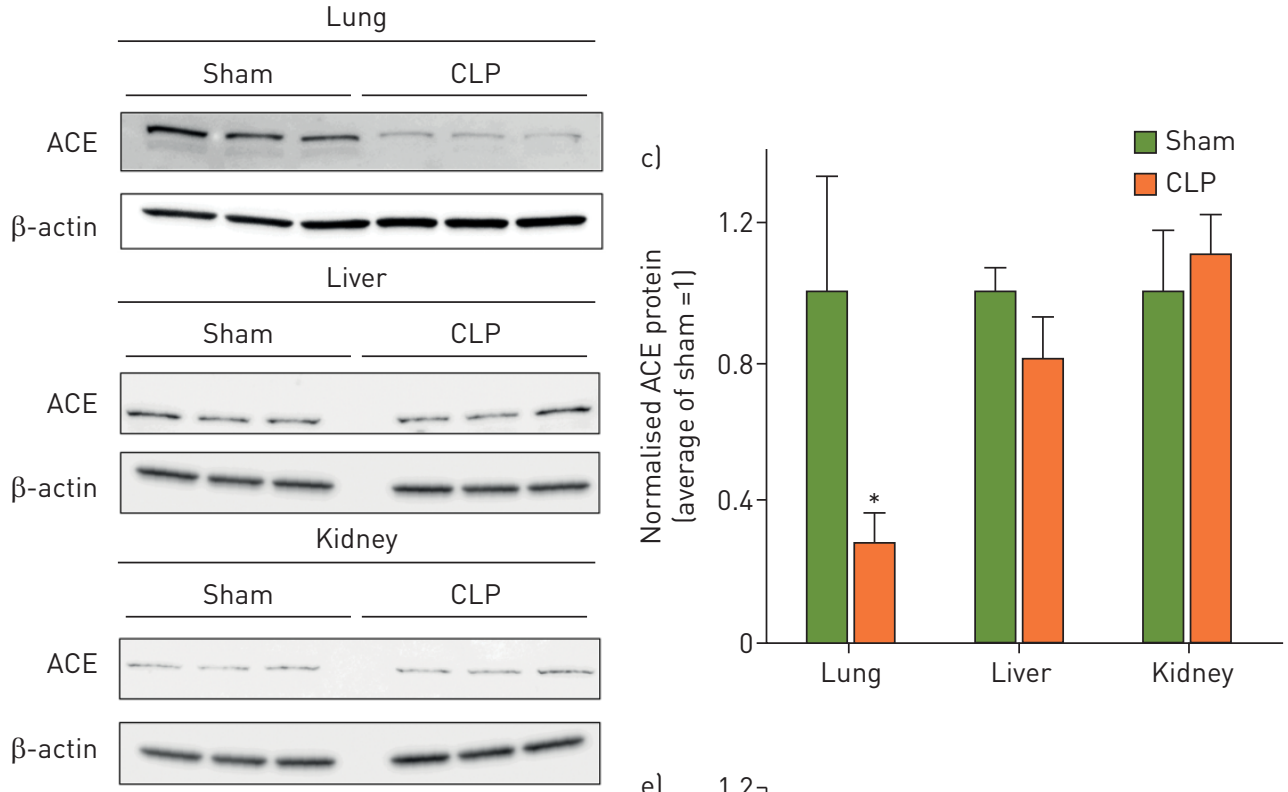

d)

VE-cadherin ${ }^{+}$ Lung endothelium
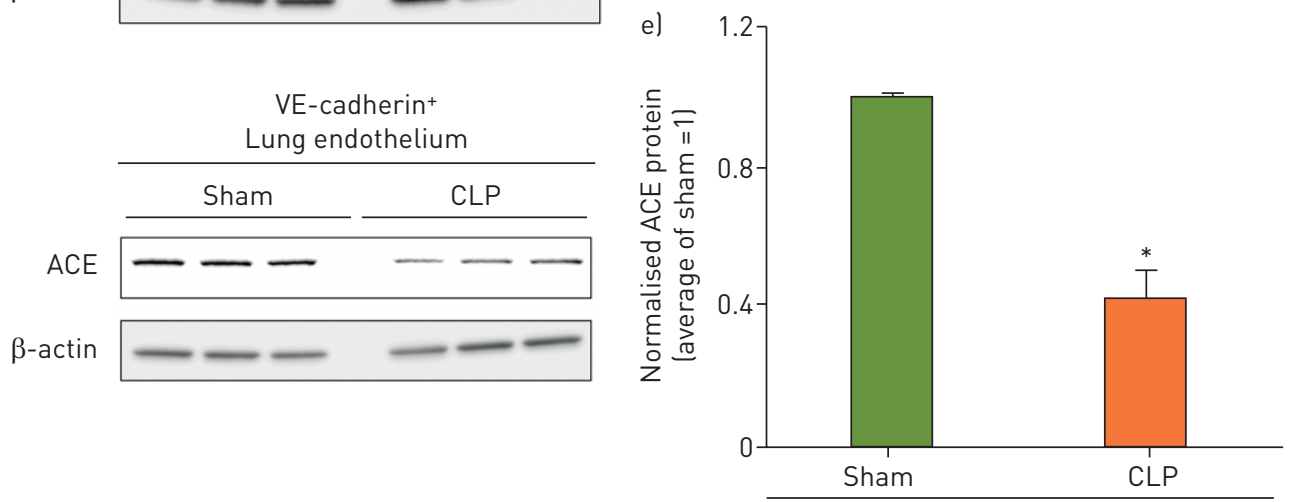

VE-cadherin ${ }^{+}$

FIGURE 4 Angiotensin-converting enzyme (ACE) protein levels decreased in the lungs of a mouse lung injury model. a) Immunohistochemical analysis for the expression of ACE in mouse alveoli. A representative image of immunohistochemical staining for ACE in a lung section of i) a sham-operated mouse or ii) a caecal ligation and puncture (CLP)-operated mouse. Scale bars=50 $\mu \mathrm{m}$. b) Representative images of Western blotting analysis for the protein levels of ACE and $\beta$-actin (endogenous control) in the homogenates of lung, liver and kidney of sham-operated mice or CLP-operated mice. c) ACE protein levels normalised by $\beta$-actin protein levels in lung, liver and kidney homogenates. $n=6$ in each group. The value is shown as a relative ratio to the mean value of sham-operated mice. Data are presented as mean \pm SD. d) An image of Western blotting analysis for the protein levels of ACE and $\beta$-actin in the cell lysates of isolated CD45- VE-cadherin ${ }^{+}$lung endothelial cells of sham-operated mice or CLP-operated mice. e) ACE protein levels normalised by $\beta$-actin protein levels in the cell lysates of lung endothelial cells. $n=3$ in each group. The value is shown as a relative ratio to the mean value of sham-operated mice. Data are presented as mean $\pm \mathrm{sD}$. ${ }^{*}: p<0.05$ between the two groups. 
VE-cadherin-positive endothelial cells from the CLP-operated mice and sham-operated mice by cell-sorting, as previously performed [31] and measured the levels of ACE protein by immunoblot. The levels of ACE protein decreased in the lung endothelial cells derived from the CLP-operated mice compared to the sham-operated control mice (figure $4 \mathrm{~d}$ and e). These data indicate that the formation of $\mathrm{ACE}^{+}$EMPs occurred during lung injury and resulted in both an increase in circulating $\mathrm{ACE}^{+} \mathrm{EMPs}$ and a decrease in ACE expression in pulmonary microvascular cells.

\section{Circulating ACE EMP numbers increased in septic patients who developed ARDS}

The animal study showed increased $\mathrm{ACE}^{+}$EMPs and their correlation with the severity of lung injury. To test the hypothesis that $\mathrm{ACE}^{+}$EMPs could serve as a prognostic indicator of ARDS development in septic patients, we examined circulating $\mathrm{ACE}^{+}$EMPs using flow cytometry (supplementary figure S6) in 82 septic patients recruited for the prospective observational study (figure 1). No CD31 ${ }^{+} \mathrm{CD} 41^{-} \mathrm{EMPs}$ in the human serum samples were detected by flow cytometry after treating these samples with Triton X-100 (supplementary figure S7a), suggesting that the serum EMPs detected by flow cytometry were lipid membrane-bound vesicles. In contrast to the EMPs detected in the culture supernatant, the CD $31^{+} \mathrm{CD} 41^{-}$ EMPs detected in serum were negative for CD9 (supplementary figure S7c). To address whether the $\mathrm{CD} 1^{+} \mathrm{CD}^{-} 1^{-}$microparticles were also derived from the leukocyte populations, a part of which also expressed CD31, we utilised CD45, a pan-leucocyte marker to distinguish leukocyte-derived microparticles. $\mathrm{CD} 31^{+} \mathrm{CD} 41^{-}$microparticles were negative for CD45 (supplementary figure S7d), suggesting that the $\mathrm{CD} 31^{+} \mathrm{CD} 41^{-}$EMPs detected in the serum were not contaminated by leukocyte-derived microparticles.

21 septic patients developed ARDS ( $\mathrm{n}=8$ direct $\mathrm{ARDS}, \mathrm{n}=13$ indirect ARDS). The patient characteristics were similar between the septic patients who developed and those who didn't develop ARDS, although the factors related to oxygenation (arterial oxygen tension/inspiratory oxygen $(\mathrm{P} / \mathrm{F})$ ratio, alveolar-arterial oxygen difference, ventilator-free days, and APACHE (Acute Physiology and Chronic Health Evaluation) II score) in the patients who developed ARDS were different from those who did not (table 1).

The counts of circulating $\mathrm{ACE}^{+}$EMPs on admission were significantly higher in the septic patients who developed ARDS than in those who did not develop ARDS (figure 5b, $\mathrm{p}<0.001$ ), but no difference was observed in the counts of total EMPs (figure 5a, $\mathrm{p}=0.843$ ). In addition, the $\mathrm{ACE}^{+} \mathrm{EMP} / \mathrm{EMP}$ ratio was significantly higher in patients who developed ARDS (figure $5 \mathrm{c}, \mathrm{p}<0.001$ ). Receiver operating characteristic analyses suggested that either the count of $\mathrm{ACE}^{+} \mathrm{EMPs}$ or the $\mathrm{ACE}^{+} \mathrm{EMP} / \mathrm{EMP}$ ratio was a possible prognostic indicator of ARDS development in septic patients (supplementary figure S8). Additionally, we analysed associations with the clinical variables in the septic patients and revealed that both $\mathrm{ACE}^{+} \mathrm{EMPs}$ and the $\mathrm{ACE}^{+} \mathrm{EMP} / \mathrm{EMP}$ ratio showed a moderate association with the $\mathrm{P} / \mathrm{F}$ ratio (table 2).

TABLE 1 The characteristics of septic patients with or without acute respiratory distress syndrome (ARDS)

\begin{tabular}{lccc} 
& Without ARDS & With ARDS & p-value \\
\hline Subjects & 61 & 21 & \\
Age years & $67(60-74)$ & $61(50-72)$ & 0.091 \\
Male & $35(57.4)$ & $13(61.9)$ & 0.408 \\
Type of sepsis & & & 0.196 \\
$\quad$ Sepsis & $25(41.0)$ & $5(23.8)$ & \\
$\quad$ Septic shock & $36(59.0)$ & $16(76.2)$ & 0.139 \\
Infection focus & & & \\
$\quad$ Pulmonary & $12(19.7)$ & $8(38.1)$ & 0.036 \\
$\quad$ Extrapulmonary & $49(80.3)$ & $13(61.9)$ & 0.073 \\
APACHE II score & $23(18-31)$ & $27(24-32)$ & 0.440 \\
Lactate mmol-L & $3.0(2.0-5.4)$ & $20(2.6-7.5)$ & $<0.001$ \\
Mechanical ventilation & $52(85.3)$ & $116(72-150)$ & $<0.001$ \\
P/F ratio & $254(146-332)$ & $455(312-589)$ & 0.001 \\
$\boldsymbol{D}_{\mathrm{A}-\text {-aO }}$ mmHg & $200(99-335)$ & $0(0-21)$ & 0.499 \\
Ventilator-free days & $21(5-25)$ & $14(6-32)$ & 0.061 \\
ICU stay days & $11(6-22)$ & $10(47.6)$ & \\
Hospital mortality & $15(24.6)$ & & \\
\hline
\end{tabular}

Data are presented as $\mathrm{n}$, median (interquartile range) or $\mathrm{n}(\%)$, unless otherwise stated. APACHE: Acute Physiology and Chronic Health Evaluation; P/F ratio: arterial oxygen tension/inspiratory oxygen ratio; $D_{\mathrm{A}-a O_{2}}$ : alveolar-arterial oxygen difference; ICU: intensive care unit. 

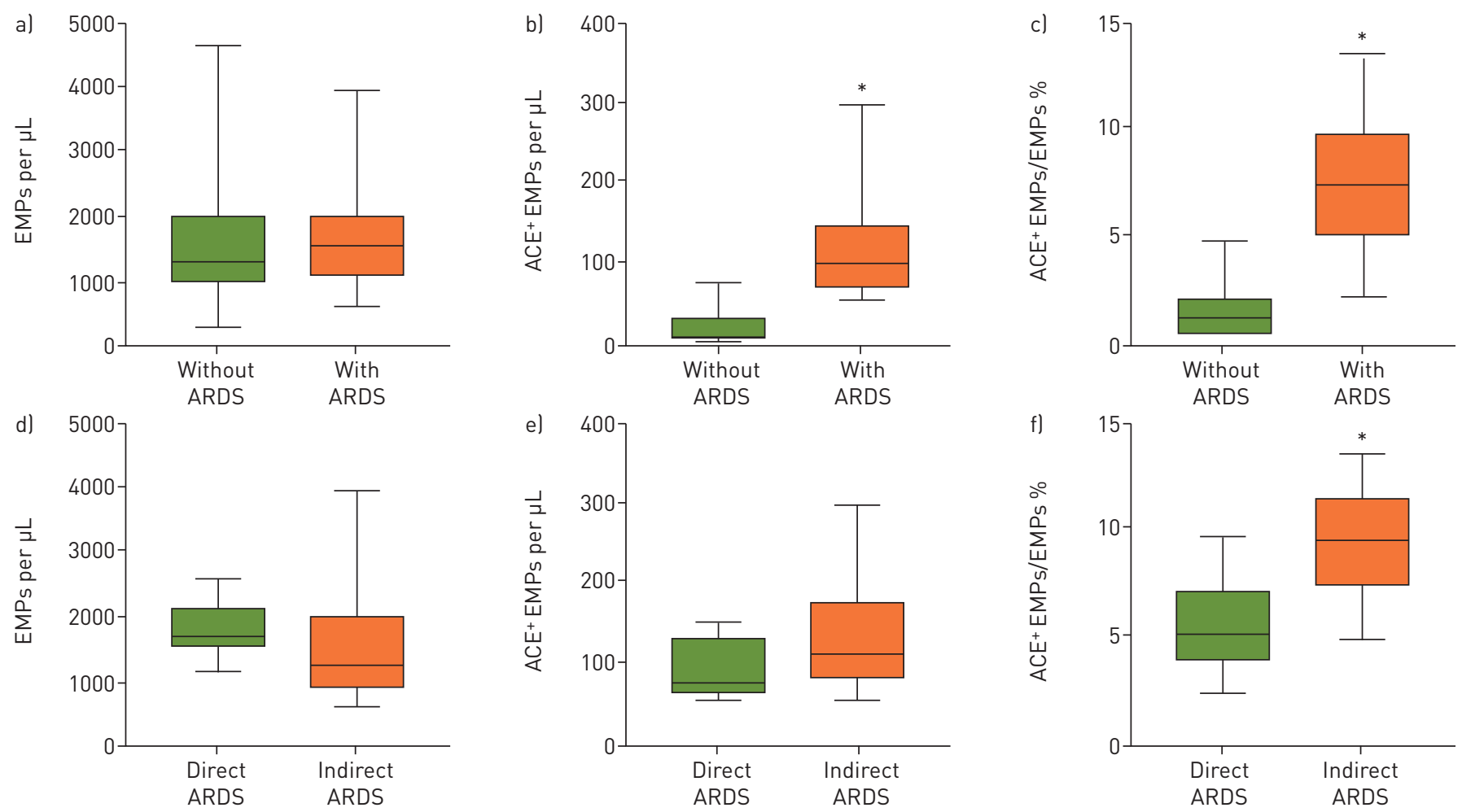

FIGURE 5 The number of circulating angiotensin-converting enzyme (ACE) ${ }^{+}$endothelial microparticles (EMPs) and the ratio of ACE ${ }^{+}$EMPs to total circulating EMPs increased in septic patients who developed acute respiratory distress syndrome (ARDS). The number of a) EMPs, b) ACE ${ }^{+}$EMPs and $c$ ) the $A C E^{+}$EMP/EMP ratio in the serum of septic patients who developed ARDS ( $n=21$ ) or who did not develop ARDS ( $n=61$ ). $d-f$ ) The ratio of $\mathrm{ACE}^{+}$EMPs to total circulating EMPs was higher in patients with indirect ARDS than in patients with direct ARDS. The number of d) EMPs, e) ACE EMPs and $f$ ) the ACE ${ }^{+}$EMP/EMP ratio in the serum of septic patients who developed direct ARDS ( $n=8$ ) or indirect $A R D S$ ( $n=13$ ). *: $p<0.05$ between the two groups.

We performed a subgroup analysis of circulating $\mathrm{ACE}^{+}$EMPs based on the source of injury for developing ARDS. The counts of total EMPs and $\mathrm{ACE}^{+}$EMPs were similar both in patients who developed direct ARDS and in those who developed indirect ARDS (figure $5 \mathrm{~d}$ and $\mathrm{e}$; $\mathrm{p}=0.130$ or 0.187 , respectively). However, the $\mathrm{ACE}^{+} \mathrm{EMP} / \mathrm{EMP}$ ratio was significantly higher in the indirect ARDS patients (figure 5f; $\mathrm{p}=0.020$ ), possibly suggesting the presence of severer pulmonary endothelial injury through indirect ARDS.

Seven septic patients developed hydrostatic pulmonary oedema on the day of admission (supplementary table S1). There was no difference of circulating EMPs in the patients that developing hydrostatic pulmonary oedema and those developing ARDS (figure 6a; $\mathrm{p}=0.843$ ). Circulating $\mathrm{ACE}^{+} \mathrm{EMPs}_{\text {and }}$ the $\mathrm{ACE}^{+} \mathrm{EMP} / \mathrm{EMP}$ ratio were significantly higher in the patients who developed ARDS than in those with hydrostatic pulmonary oedema (figure $6 \mathrm{~b}$ and $\mathrm{c} ; \mathrm{p}<0.001$ ).

TABLE 2 Association between the clinical variables and the counts of serum endothelial microparticles (EMPs), angiotensin-converting enzyme (ACE) ${ }^{+}$EMPs or the ACE ${ }^{+} \mathrm{EMP} / \mathrm{EMP}$ ratio in the septic patients

\begin{tabular}{|c|c|c|c|c|c|c|}
\hline & \multicolumn{2}{|c|}{ EMPs per $\mu \mathrm{L}$} & \multicolumn{2}{|c|}{$\mathrm{ACE}^{+}$EMPs per $\mu \mathrm{L}$} & \multicolumn{2}{|c|}{$\mathrm{ACE}^{+} \mathrm{EMP} / \mathrm{EMP}$ ratio $\%$} \\
\hline & $r_{s}$ & p-value & $r_{s}$ & p-value & $r_{s}$ & p-value \\
\hline Age & -0.1103 & 0.3239 & -0.1542 & 0.1666 & -0.1867 & 0.093 \\
\hline APACHE II score & 0.0197 & 0.8609 & 0.1338 & 0.2307 & 0.105 & 0.3477 \\
\hline Lactate $\mathrm{mmol} \cdot \mathrm{L}^{-1}$ & -0.0209 & 0.852 & 0.2058 & 0.0636 & 0.1841 & 0.0978 \\
\hline $\mathrm{P} / \mathrm{F}$ ratio & -0.0628 & 0.5753 & -0.3954 & 0.0002 & -0.3319 & 0.0023 \\
\hline
\end{tabular}

The correlations were calculated using the Spearman rank correlation test. $r_{s}$ : Spearman rank correlation coefficient; APACHE: Acute Physiology and Chronic Health Evaluation; P/F ratio: arterial oxygen tension/ inspiratory oxygen ratio. 

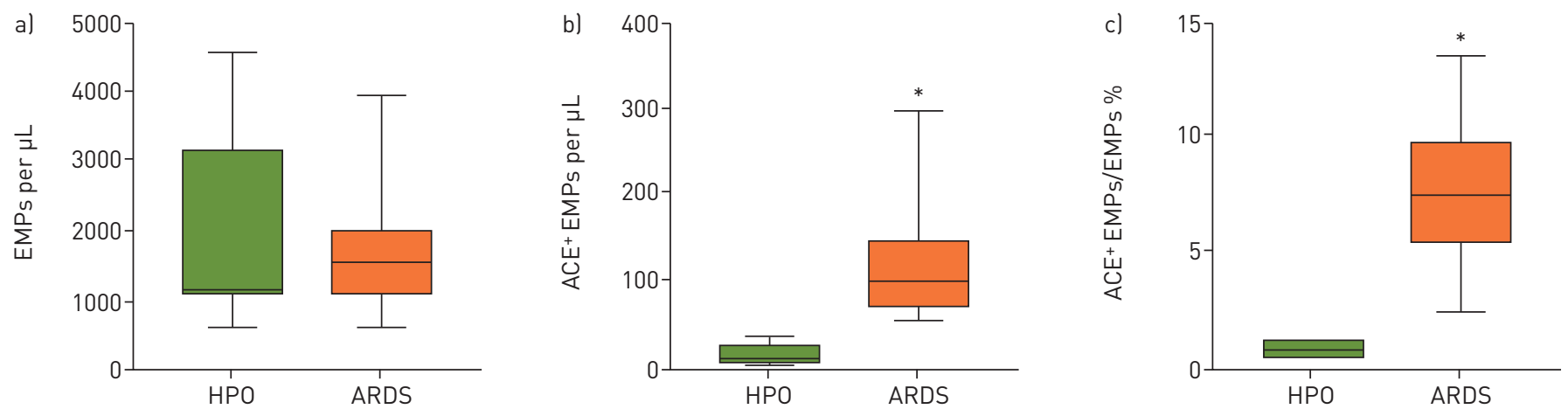

FIGURE 6 The counts of circulating angiotensin-converting enzyme (ACE) ${ }^{+}$endothelial microparticles (EMPs) and the ratio of ACE $^{+}$EMPs to total circulating EMPs was higher in septic patients with acute respiratory distress syndrome (ARDS) (non-hydrostatic pulmonary oedema (HPO)) than in patients with HPO. The number of a) EMPs, b) ACE ${ }^{+}$EMPs and c) the ACE ${ }^{+}$EMP/EMP ratio in the serum of septic patients who developed HPO $(n=7)$ or ARDS $(n=21) .{ }^{*}$ : $p<0.05$ between the two groups.

10 out of 21 septic patients who developed ARDS died in hospital (table 1). There was no significant difference in any of the three EMP parameters between survivors and non-survivors (supplementary figure S9). Logistic regression analyses suggested that the APACHE II score was a significant prognostic factor for in-hospital mortality (supplementary table S2).

For the time-course analysis, we could collect serum samples continuously until day 7 from 10 ARDS patients. The numbers of total EMPs remained unchanged during the observational period (supplementary figure S10a). $\mathrm{ACE}^{+}$EMPs significantly decreased on day 5, and the $\mathrm{ACE}^{+} \mathrm{EMP} / \mathrm{EMP}$ ratio significantly decreased on day 5 and 7 compared with day 1 (supplementary figure S10b and c). Four patients died in hospital. There were no significant differences except that the $\mathrm{ACE}^{+} \mathrm{EMP} / \mathrm{EMP}$ ratio decreased on day 7 only in survivors (supplementary figure S11).

\section{Discussion}

In this study, we show that inflammatory stimuli induced the formation of $\mathrm{ACE}^{+} \mathrm{EMPs}$ (EMPs highly expressing ACE) from pulmonary microvascular endothelial cells, and the activation of caspases and apoptosis may be partially involved in this process. In mouse lung injury models, circulating $\mathrm{ACE}^{+} \mathrm{EMPs}$ were significantly increased and the $\mathrm{ACE}^{+}$EMP numbers correlated with the W/D lung ratio, one of the indications for alterations in the alveolar-capillary barrier. The prospective cohort study of the septic patients showed that the $\mathrm{ACE}^{+} \mathrm{EMPs}$ and the $\mathrm{ACE}^{+} \mathrm{EMP} / \mathrm{EMP}$ ratio on admission were significantly higher in the septic patients who developed ARDS. These data suggest that $\mathrm{ACE}^{+} \mathrm{EMPs}$ may reflect the injury of the lung microvasculature and could be a prognostic marker for the development of ARDS in septic patients.

Our study using cultured cells revealed that lung microvascular endothelial cells release more $\mathrm{ACE}^{+} \mathrm{EMPs}$ than systemic microvascular endothelial cells. Therefore, we focused on EMPs highly expressing ACE to distinguish lung microvascular EMPs from other EMPs for a more precise evaluation of lung endothelial injury. In both the CLP-induced lung injury model and LPS-induced lung injury model, the counts of both total circulating EMPs and $\mathrm{ACE}^{+}$EMPs and the $\mathrm{ACE}^{+} \mathrm{EMP} / \mathrm{EMP}$ ratio increased compared with the controls. The immunohistochemical analyses showed a decrease in the intensity of ACE staining in the CLP-induced lung injury mice. These data suggest that inflammation induces the formation of microparticles from lung microvascular endothelial cells, resulting in an increase in the circulating $\mathrm{ACE}^{+}$ EMP numbers during lung injury. Our data from the mouse models also revealed a correlation between circulating $\mathrm{ACE}^{+} \mathrm{EMPs}$ and the W/D lung ratio, suggesting that $\mathrm{ACE}^{+}$EMPs could reflect the severity of lung injury including alterations in the alveolar-capillary barrier during acute lung injury.

Our clinical study showed that both parameters, circulating $\mathrm{ACE}^{+} \mathrm{EMPs}$ and the $\mathrm{ACE}^{+} \mathrm{EMP} / \mathrm{EMP}$ ratio on admission, significantly increased in septic patients who developed ARDS compared to those who did not. Moreover, these $\mathrm{ACE}^{+} \mathrm{EMP}$ factors were also significantly higher in patients with ARDS compared to the patients with hydrostatic oedema. These observations may suggest that $\mathrm{ACE}^{+} \mathrm{EMPs}$ could be a prognostic marker for the development of ARDS in susceptible patients such as septic patients and also for distinguishing ARDS from hydrostatic pulmonary oedema.

The $\mathrm{ACE}^{+} \mathrm{EMP} / \mathrm{EMP}$ ratio was significantly higher in patients with indirect ARDS than in patients with direct ARDS, suggesting that pulmonary endothelial injury could be more severe in indirect ARDS than in direct ARDS. The mechanisms of the disruption of the alveolar-capillary barrier in direct and indirect 
ARDS are probably different. Animal models suggest that epithelial injury is greater in direct ARDS [32], but endothelial injury is dominant in indirect ARDS [33]. Previous clinical studies also showed that direct ARDS patients showed an increase in circulating surfactant protein $\mathrm{D}$ and soluble receptor for advanced glycation end-product as markers of pulmonary epithelial injury, but indirect ARDS patients showed an increase in angiopoietin-2 as a marker of endothelial injury [34]. These previously reported data were consistent with the findings of our study.

There are limitations in this study. First, our data suggest that $\mathrm{ACE}^{+} \mathrm{EMPs}$ and the $\mathrm{ACE}^{+} \mathrm{EMP} / \mathrm{EMP}$ ratio may not be a predictive marker for mortality, whereas APACHE II could be predictive. This may suggest that the severity of multi-organ failure could be a better predictor than the severity of pulmonary vascular injury during sepsis-induced ARDS. Second, although our findings from the experiments with a lipid-solubilising detergent may suggest that the EMPs detected in our studies were lipid membrane-bound vesicles, we could not perform further morphological characterisation by electron microscopic analysis for $\mathrm{ACE}^{+}$EMPs detected by flow cytometry in serum samples, because we could not succeed in collecting EMPs or $\mathrm{ACE}^{+}$EMPs by the flow-cytometric cell sorter. We also could not reveal the physiological and pathological roles of $\mathrm{ACE}^{+}$EMPs in ARDS patients because we have not established isolated EMPs in serum samples. We tried to analyse the contents of the total extracellular vesicles including microparticles; however, we could not analyse the contents in detail including mRNA of inflammatory cytokines in the extracellular vesicles because we did not have sufficient amounts of the clinical samples. The development of techniques to isolate specific microparticles, including EMPs, would be helpful for investigating the roles of EMPs in both physiological and pathological conditions. Third, our clinical study was conducted in a single centre and the sample size was relatively small. To confirm the significance of $\mathrm{ACE}^{+} \mathrm{EMPs}_{\mathrm{s}}$ a predictive or diagnostic marker for ARDS, we will need validation studies with more subjects. Fourth, although our study and previous reports showed that the levels of ACE expression were significantly higher in pulmonary microvascular endothelial cells than in microvascular endothelial cells from other organs [20] and pulmonary artery endothelial cells (supplementary figure S1a and b), ACE is not an optimal specific marker to distinguish pulmonary microvascular endothelial cells from other endothelial cells including systemic microvascular endothelial cells. Therefore, there might be a possibility that circulating $\mathrm{ACE}^{+}$EMPs detected in serum contains EMPs derived from other endothelial cells. However, our in vitro study also showed that the expression levels of ACE on EMPs derived from pulmonary microvascular endothelial cells were significantly higher than on those from systemic microvascular endothelial cells (figure 2e), suggesting that, although ACE is not a specific marker for pulmonary microvascular endothelium and its EMPs, EMPs expressing higher levels of ACE could be derived from pulmonary microvascular endothelium.

In conclusion, the results of our study suggest that $\mathrm{ACE}^{+}$EMPs from pulmonary microvascular endothelial cells may reflect the injury of pulmonary microvascular endothelial cells, and could be a prognostic marker for developing ARDS in septic patients as well as a diagnostic marker for distinguishing ARDS from hydrostatic pulmonary oedema.

Acknowledgements: We thank Brent K. Bell (Tohoku University Graduate School of Medicine, Sendai, Japan) for critical reading of the manuscript and language assistance. We acknowledge the support of the Biomedical Research Unit of Tohoku University Hospital. We thank M. Kondo (Tohoku University Graduate School of Medicine, Sendai, Japan) for clerical support.

Conflict of interest: Y. Takei has nothing to disclose. M. Yamada reports grants from Japan Society for the Promotion of Science, during the conduct of the study; grants from Novartis, personal fees for lectures from Pfizer, Meiji Seika Pharma and AstraZeneca, outside the submitted work. Koji Saito reports grants from Japan Society for the Promotion of Science, during the conduct of the study. Y. Kameyama has nothing to disclose. H. Sugiura reports grants from Japan Society for the Promotion of Science, outside the submitted work. T. Makiguchi reports grants from Japan Society for the Promotion of Science, outside the submitted work. N. Fujino reports grants from Japan Society for the Promotion of Science, outside the submitted work. A. Koarai reports grants from Japan Society for the Promotion of Science, Novartis and Terumo Foundation for Life Sciences and Arts, outside the submitted work. H. Toyama reports grants from Japan Society for the Promotion of Science, outside the submitted work. Kazutomo Saito reports grants from Japan Society for the Promotion of Science, outside the submitted work. Y. Ejima has nothing to disclose. Y. Kawazoe has nothing to disclose. D. Kudo reports personal fees for lectures from Asahikasei Pharma, Japan Blood Products Organization and Sumitomo Dainippon Pharma, outside the submitted work. S. Kushimoto has nothing to disclose. M. Yamauchi reports grants from Japan Society for the Promotion of Science and Japan Agency for Medical Research and Development, outside the submitted work. M. Ichinose reports personal fees for lectures from Boehringer Ingelheim and AstraZeneca, grants from Astellas, grants from Shionogi, Pfizer, Daiichi Sankyo, Boehringer Ingelheim, Taiho Pharmaceutical Co., AstraZeneca, Fukuda Denshi, Japan Society for the Promotion of Science and Japan Agency for Medical Research and Development, outside the submitted work.

Support statement: This work was supported by Grants-in-Aid for Scientific Research (15K15562 and 18K08907 to K. Saito, and 15K09206 and 18K08134 to M. Yamada) from the Japan Society for the Promotion of Science (JSPS). Funding information for this article has been deposited with the Crossref Funder Registry. 


\section{References}

1 Burger D, Schock S, Thompson CS, et al. Microparticles: biomarkers and beyond. Clin Sci 2013; 124: 423-441.

2 Meziani F, Delabranche X, Asfar P, et al. Bench-to-bedside review: circulating microparticles - a new player in sepsis? Crit Care 2010; 14: 236.

3 Schiro A, Wilkinson FL, Weston R, et al. Endothelial microparticles as conveyors of information in atherosclerotic disease. Atherosclerosis 2014; 234: 295-302.

4 Yun JW, Xiao A, Tsunoda I, et al. From trash to treasure: the untapped potential of endothelial microparticles in neurovascular diseases. Pathophysiology 2016; 23: 265-274.

5 Nie DM, Wu QL, Zheng P, et al. Endothelial microparticles carrying hedgehog-interacting protein induce continuous endothelial damage in the pathogenesis of acute graft-versus-host disease. Am J Physiol Cell Physiol 2016; 310: C821-C835.

6 Amabile N, Guérin AP, Leroyer A, et al. Circulating endothelial microparticles are associated with vascular dysfunction in patients with end-stage renal failure. J Am Soc Nephrol 2005; 16: 3381-3388.

7 Faure V, Dou L, Sabatier F, et al. Elevation of circulating endothelial microparticles in patients with chronic renal failure. J Thromb Haemost 2006; 4: 566-573.

8 Feng B, Chen Y, Luo Y, et al. Circulating level of microparticles and their correlation with arterial elasticity and endothelium-dependent dilation in patients with type 2 diabetes mellitus. Atherosclerosis 2010; 208: 264-269.

9 Gordon C, Gudi K, Krause A, et al. Circulating endothelial microparticles as a measure of early lung destruction in cigarette smokers. Am J Respir Crit Care Med 2011; 184: 224-232.

10 Jung C, Sörensson P, Saleh N, et al. Circulating endothelial and platelet derived microparticles reflect the size of myocardium at risk in patients with ST-elevation myocardial infarction. Atherosclerosis 2012; 221: $226-231$.

11 Nozaki T, Sugiyama S, Koga H, et al. Significance of a multiple biomarkers strategy including endothelial dysfunction to improve risk stratification for cardiovascular events in patients at high risk for coronary heart disease. J Am Coll Cardiol 2009; 54: 601-608.

12 Takahashi T, Kobayashi S, Fujino N, et al. Increased circulating endothelial microparticles in COPD patients: a potential biomarker for COPD exacerbation susceptibility. Thorax 2012; 67: 1067-1074.

13 ARDS Definition Task Force. Acute respiratory distress syndrome: the Berlin definition. JAMA 2012; 307: 2526-2533.

14 Ware LB, Matthay MA. The acute respiratory distress syndrome. N Engl J Med 2000; 342: 1334-1349.

15 Bhattacharya J, Matthay MA. Regulation and repair of the alveolar-capillary barrier in acute lung injury. Annu Rev Physiol 2013; 75: 593-615.

16 Matthay MA, Zimmerman GA. Acute lung injury and the acute respiratory distress syndrome: four decades of inquiry into pathogenesis and rational management. Am J Respir Cell Mol Biol 2005; 33: 319-327.

17 Coates D. The angiotensin converting enzyme (ACE). Int J Biochem Cell Biol 2003; 35: 769-773.

18 Aird WC. Phenotypic heterogeneity of the endothelium: II. Representative vascular beds. Circ Res 2007; 100: $174-190$

19 Danilov S, Jaspard E, Churakova T, et al. Structure-function analysis of angiotensin I-converting enzyme using monoclonal antibodies. Selective inhibition of the amino-terminal active site. J Biol Chem 1994; 269: 26806-26814.

20 Danilov SM, Gavrilyuk VD, Franke FE, et al. Lung uptake of antibodies to endothelial antigens: key determinants of vascular immunotargeting. Am J Physiol Lung Cell Mol Physiol 2001; 280: L1335-L1347.

21 Razavi HM, Werhun R, Scott JA, et al. Effects of inhaled nitric oxide in a mouse model of sepsis-induced acute lung injury. Crit Care Med 2002; 30: 868-873.

22 Tanaka KI, Tamura F, Sugizaki T, et al. Evaluation of lecithinized superoxide dismutase for the prevention of acute respiratory distress syndrome in animal models. Am J Respir Cell Mol Biol 2017; 56: 179-190.

23 Yamada M, Gomez JC, Chugh PE, et al. Interferon- $\gamma$ production by neutrophils during bacterial pneumonia in mice. Am J Respir Crit Care Med 2011; 183: 1391-1401.

24 Zhu Z, Sun L, Hao R, et al. Nedd8 modification of Cullin-5 regulates lipopolysaccharide-induced acute lung injury. Am J Physiol Lung Cell Mol Physiol 2017; 313: L104-L114.

25 Matute-Bello G, Downey G, Moore BB, et al. An official American Thoracic Society workshop report: features and measurements of experimental acute lung injury in animals. Am J Respir Cell Mol Biol 2011; 44: 725-738.

26 Lan CC, Peng CK, Tang SE, et al. Inhibition of Na-K-Cl cotransporter isoform 1 reduces lung injury induced by ischemia-reperfusion. J Thorac Cardiovasc Surg 2017; 153: 206-215.

27 Singer M, Deutschman CS, Seymour CW, et al. The Third International Consensus Definitions for Sepsis and Septic Shock (Sepsis-3). JAMA 2016; 315: 801-810.

28 Sánchez-López V, Vila-Liante V, Arellano-Orden E, et al. High correlation between 2 flow cytometry platforms in the microparticles analysis using a new calibrated beads strategy. Transl Res 2015; 166: 733-739.

29 Cheng KT, Xiong S, Ye Z, et al. Caspase-11-mediated endothelial pyroptosis underlies endotoxemia-induced lung injury. J Clin Invest 2017; 127: 4124-4135.

30 Curtis AM, Wilkinson PF, Gui M, et al. p38 mitogen-activated protein kinase targets the production of proinflammatory endothelial microparticles. J Thromb Haemost 2009; 7: 701-709.

31 Yamada M, Kubo H, Ota C, et al. The increase of microRNA-21 during lung fibrosis and its contribution to epithelial-mesenchymal transition in pulmonary epithelial cells. Respir Res 2013; 14: 95.

32 Menezes SL, Bozza PT, Neto HC, et al. Pulmonary and extrapulmonary acute lung injury: inflammatory and ultrastructural analyses. J Appl Physiol 2005; 98: 1777-1783.

33 Pelosi P, D’Onofrio D, Chiumello D, et al. Pulmonary and extrapulmonary acute respiratory distress syndrome are different. Eur Respir J 2003; 22: Suppl. 42, 48s-56s.

34 Calfee CS, Janz DR, Bernard GR, et al. Distinct molecular phenotypes of direct vs indirect ARDS in single-center and multicenter studies. Chest 2015; 147: 1539-1548. 